Chapter 8

\title{
Autophagy: A Possible Defense Mechanism in Parkinson's Disease?
}

\author{
Rosa A. González-Polo, Rubén Gómez-Sánchez, \\ Lydia Sánchez-Erviti, José M Bravo-San Pedro, \\ Elisa Pizarro-Estrella, Mireia Niso-Santano and \\ José M. Fuentes
}

Additional information is available at the end of the chapter

http://dx.doi.org/10.5772/54851

\section{Introduction}

Growing evidence supports an active role for deregulated macroautophagy (autophagic stress) in neuronal cell death and neurodegenerative diseases, as Parkinson's disease (PD). The exact etiology of PD is currently unknown, but it seems clear that its pathogenesis is a multifactorial process. The detection of genetic alterations may be useful as a biomarker of early molecular diagnosis, but it is also important to know and identify changes at the molecular level, more frequent in cases of Parkinsonism. In this sense, the phenomenon of autophagic cell death, described in the normal nervous system, could be the result of a pathological process, such as those related to neurodegenerative diseases. Autophagy is an intracellular catabolic mechanism mediated by lysosomes, which is responsible for most of the degradation and recycling of cytoplasmic components and intracellular organelles dysfunctional or damaged. For a long time, it is unknown why the aggregation of proteins and developmental neurotoxicity are given in a later period of life, even in familial forms of the disease, where the mutant protein is present throughout life in the individual. It has been shown that genetic ablation of autophagy induces neurodegeneration and accumulation of ubiquitinated proteins. In addition, some genetic mutations that cause neurodegenerative diseases directly affect proteolytic systems responsible for the degradation of the mutant protein. In this paper, we analyze the possible neuroprotective role that autophagy-inducing substances can have on the mechanism and development of PD. 


\section{Contents}

\subsection{The role of autophagy in PD - Importance of oxidative stress}

PD is a progressive neurodegenerative disorder characterized by slow movements (bradykinesia), poverty of movements (hypokinesia), resting tremor and rigidity. PD is characterized pathologically by the loss of dopaminergic neurons in the substantia nigra pars compacta and the presence of Lewy bodies (LB), which are intra-cytoplasmic inclusions containing high levels of alpha-synuclein protein. Combined with the ubiquitous alphasynuclein aggregates in PD brains the finding of mutations and multiplications of the alpha-synuclein gene in familial forms of PD add to the importance of alpha-synuclein to PD pathology. Recent observations have suggested that alpha-synuclein can be directly transmitted from pathological affected neurons to healthy unaffected neurons. This is consistent with the Braak staging of PD pathology which suggests there is a progression of LB pathology from the brainstem to the cortex consistent with the pathological propagation along specific neural pathways. The release of alpha-synuclein from cells and the transfer of an aggregated form of alpha-synuclein to neighboring cells have been demonstrated in vitro and in vivo and is potentially an important step in the progression of PD pathology. While these processes are poorly understood, exosomes have been associated with the release and transfer of prion protein via a novel processing pathway that involves the N-terminal modification of PrP and selection of distinct PrP glycoforms for incorporation into these vesicles and there is increasing evidence that alpha-synuclein is also released from cells in exosomes. Lysosomal dysfunction increases alpha-synuclein release and transmission mediated by exosomes in cell models.

While the primary cause of PD in the majority of patients is not known, the number of genetic causes and risk factors are gradually increasing and are beginning to highlight important pathogenetic pathways. The role of mitochondrial dysfunction in PD is supported by the ability of the mitochondrial toxins MPTP (1-methyl-4-phenylpyridinium) and rotenone to target the dopaminergic neurons and more recently by PTEN-induced kinase 1 (PINK1) and parkin, which are mutated in PD and have a role in regulating mitochondrial integrity. Oxidative stress and damage are important observations in PD brains and are common observations in various genetic and toxin models of PD. Protein turnover pathways have been implicated in PD, initially involving the proteasomal system and more recently autophagy and the lysosomal pathways. The G2019S mutation of leucine-rich repeat kinase 2 (LRRK2) is a relatively common cause of PD, and recessive mutations of the lysosomal enzyme glucocerebrosidase-1 (GBA-1) have recently been identified as one of the most important genetic risk factors for PD although the disease mechanisms have not yet been elucidated.

Autophagy is a catabolic pathway for destruction and turnover of long-live proteins and organelles in lysosomes. Autophagy contributes to degradation of damaged long-live proteins and organelles and the normal turn-over of these components, moreover is up-regulated in response to external stressors as starvation and oxidative stress. In mammalian cells autophagy comprises three separate pathways: macroautophagy, microautophagy and chaperone-mediated autophagy (CMA). 
Macroautophagy involves the de novo formation of double membrane vacuoles, autophagosomes originated from mitochondria and/or plasma membrane. Autophagosomes fuse with lysosomes to deliver cytoplasmic contents including misfolded or aggregated proteins and organelles for digestion and recycling of amino acids. Macroautophagy is regulated at molecular level by proteins of the Atg family, which form dynamic complexes involved in the assembly, docking and degradation of the autophagosome. The serine/threonine kinase mTOR (mammalian target of rapamicyn) is a key mediator of macroautophagy upregulation under starvation, this protein regulates cell cycle progression, protein synthesis and cell growth. Activated mTOR promotes protein synthesis and inhibits catabolism by decreasing macroautophagy in a mechanism mediated by Atg13 phosphorylation that modulates Atg1 activity. During starvation mTOR is inactivated resulting in hypophosphorylation of Atg13 and stimulation of Atg1-Atg13 complexes required for induction of autophagy.

Microautophagy involves lysosomal pinocytosis of cytoplasmic contents and is involved in the turnover of long half-life cytosolic proteins. However, the underlying process is poorly understood in mammalian cells.

It is important to note that both macro and microautophagy are highly conserved process that occurs in same manner in all cells. In this sense most of the knowledge of autophagy was first described in yeast.

CMA is a selective autophagic pathway dependent upon the protein chaperone hsc70 and its binding to LAMP-2A (Lysosomal-associated membrane protein 2A), a lysosomal surface receptor. A highly specific subset of cytosolic proteins with a KFREQ motif are recognized by the hsc70 chaperone and internalized for degradation by Lysosomal-associated membrane protein 2A LAMP-2A lysosomal membrane receptors. The substrate protein is unfolded by the complex and is translocated to the lysosome with the help of a lysosomal chaperone (lys-hsc70).

Recent reports identified a increase in autophagic vacuoles, decrease in macroautophagy and CMA proteins in PD brain areas affected by the neurodegenerative process, which might have a direct impact in alpha-synuclein levels as this protein in degraded in lysosomes mainly by CMA. The increase cytosolic alpha-synuclein levels favours the modification of alpha-synuclein and formation of oligomers, fibrils and aggregates. Indeed, CMA is important in the removal of oxidized and altered proteins during conditions of mild oxidative stress, and blockage of CMA resulted in increased intracellular levels of oxidized and aggregate proteins. Conversely the pathological environment in PD may lead to alpha-synuclein post-translational modifications (e.g. nitration, oxidation, dopamine adducts), or oligomeric or fibrilar formations which may not be degraded by CMA and may even inhibit CMA promoting aggregation. Moreover, mutations in PINK1 and parkin proteins, associated with PD, affect the remove of damaged mitochondria by lysosomes (mitophagy). Finally, other genetic mutations associated with PD, as ATP13A2 and LRRK2, affect autophagy and lysosomal function, reinforcing the involvement of autophagic function in PD.

Several studies described an increase in markers related with oxidative stress as protein nitration and lipid peroxidation in LB. Additional findings described a decrease in several de- 
fense antioxidant molecules in PD including the thiol-reducing agent glutathione (GSH) and the glutathione peroxidase activity. This increase in reactive oxygen species (ROS) levels involves an increased oxidized alteration of proteins as alpha-synuclein resulting in increasing protein misfolding and impaired degradation, which might cause the accumulation of toxic soluble oligomers or insoluble aggregates.

Mitochondria are the main source of ROS involved in oxidative stress and its role in PD is highlighted by the toxic animal models (MPTP, 6-hydroxydopamine (6-OHDA), rotenone) and the mutation in PINK1, parkin and DJ-1, involved in mitochondrial function and turnover, is related with early onset PD. When oxidative processes clearly contribute to the pathology and progression of PD the initiation of this cascade is probably secondary to other causes.

\subsection{Toxic stress inducers related to PD}

MPTP. In the early 1980s, a group of young drug abusers from Northern California developed a parkinsonian syndrome clinically indistinguishable from PD caused by the intravenous injection of a synthetic analog of demerol. Chemical analysis of this synthetic drug showed that it contained around 3\% of MPTP. Post-mortem investigations clearly confirm the lesion of the substantia nigra MPTP was thus considered as a powerful drug to induce nigral degeneration both rodent and primate models of PD. Since that time, a number of environmental toxicants have been associated with PD etiology including metals, solvents, and pesticides. Epidemiologic and toxicological studies suggest a consistent correlation between pesticide exposure and PD. MPTP is a lipophilic toxin that rapidly crosses the blood-brain barrier. Inside the brain, MPTP is oxidized to an unstable intermediary 1-methyl-4-phenyl-2,3-dihydroxipyridinium $\left(\mathrm{MPDP}^{+}\right)$via the action of monoamine oxidase $\mathrm{B}$ (MAO-B). MPDP then undergoes spontaneous oxidation to the active toxic 1-methyl-4-phenylpiridinium $\left(\mathrm{MPP}^{+}\right)$. Following its release into the extracellular space, $\mathrm{MPP}^{+}$is taken up by the dopamine transporter (DAT), the amino acid transporter cationic or glutamate transporter. Once inside the cell, cytoplasmatic $\mathrm{MPP}^{+} \mathrm{can}$ trigger the production of ROS, which may contribute to its overall neurotoxicity. However, the majority of $\mathrm{MPP}^{+}$is transported into the mitochondria of dopaminergic neurons, where it disrupts oxidative phosphorylation by inhibiting complex I activity. The inhibition of mitochondrial electron transport chain causes an acute ATP deficiency, loss of mitochondrial membrane potential, alterations of intracellular calcium levels and increase ROS production, particularly superoxide. Finally, $\mathrm{MPP}^{+}$induces the activation of cell death signaling pathways such as c-jun N-terminal kinase (JNK), p38 mitogen activated kinase and Bax Moreover MPTP interact with familial PD gene products. It has reported that MPTP triggers an upregulation of alpha-synuclein and is accompanied by PD-like modifications of alpha-synuclein, including its aggregation, nitration and phosphorylation and modifies parkin solubility causing Parkin aggregation.

Rotenone. Rotenone is an non mutagenic active agent of many pesticides and a well-characterized, high affinity specific inhibitor of complex I of the mitochondrial respiratory chain which emerged in the $90^{\prime}$ s as a potential parkinsonian environmental toxin. Because it is highly lipophilic, it crosses biological membranes easily and apparently independent of specific transport system (unlike $\mathrm{MPP}^{+}$), and it gets into the brain very quickly, where accumulates within 
mitochondria and inhibits complex I. Despite this complex I inhibition, rotenone caused selective degeneration of the nigrostriatal dopaminergic pathway, selective striatal oxidative damage, and formation of ubiquitin- and alpha-synuclein positive inclusions in nigral cells, which were similar to the LB of PD. Furthermore, rotenone activates both mitochondrial and endoplasmic dependent caspases that induces apoptosis. Additionally, increased oxidative stress, ubiquitin accumulation, proteasomal inhibition and inflammation, all have been observed in response to rotenone exposure. Rotenone has also been reported to induce caspase independent cell death, accumulation of alpha-synuclein, parkin aggregation and oxidation mitochondrial of thioredoxin. Rotenone and MPTP share the same principle mechanism of action and their toxic effects are quite similar. The toxicity of both compounds is linked with glial cell activation. Microglial activation is exhibited in the rotenone rat model of Parkinsonism, occurring prior to evidence of dopaminergic cell loss.

6-OHDA. 6-OHDA was the first chemical compound discovered with specific neurotoxic effects on catecholaminergic pathways. 6-OHDA is one of the most common neurotoxins used to experimentally model nigral degeneration in vitro as well as in vivo. 6-OHDA is a hydroxylate analogue of the natural dopamine that induces degeneration of dopaminergic neurons in the nigrostriatal tract and is commonly used to model dopaminergic degeneration both in vitro and in vivo. 6-OHDA is taken up into dopaminergic neurons via the dopamine transporter although it shows high affinity for the noradrenaline transporter. Consequently, 6-OHDA can produce specific degeneration of catecholaminergic neurons. Interestingly, 6-OHDA can be formed from dopamine by non-enzymatic hydroxylation in presence of $\mathrm{Fe}^{2+}$ and $\mathrm{H}_{2} \mathrm{O}_{2}$. The mechanism of action of 6-OHDA is related to its pro-oxidant properties. Once in the neuron, 6-OHDA accumulates in the cytosol and readily oxidizes to form ROS, mostly hydrogen peroxide and paraquinone to reduce striatal levels of antioxidant enzymes and to elevate levels of iron in the system nervous. As an additional mechanism, 6-OHDA can accumulate in the mitochondria where interacts directly with complexes I and IV of the mitochondrial respiratory chain causing respiratory inhibition and oxidative stress. Moreover, oxidized proteins by 6-OHDA induce ER stress and upregulation of the unfolded protein response (UPR), which regulates protein translation, protein folding and protein degradation. Like dopamine and other related compounds, 6-OHDA can be metabolized by monoamine oxidase to generate ROS. However, pretreatment with MAO inhibitors enhances the toxicity of 6-OHDA, offering evidence against the contributions of MAO-dependent ROS sources in the neurotoxic process. The 6-OHDA model has been extensively used ever since and is still the most widely used tool for replicating a PD-like loss of dopaminergic neurons in the substantia nigra pars compacta.

Paraquat. The potent herbicide paraquat ( $\mathrm{PQ}, \mathrm{N}, \mathrm{N}^{\prime}$-dimethyl-1,4,4'-bipiridinium) was first identified as a prototypic non mutagenic neurotoxin due to shares structural similarities to $\mathrm{MPP}^{+}$, the active metabolite of MPTP. PQ is highly effective, fast-acting and non-selective herbicide widely used herbicide in the world. PQ has been banned or restricted by Environmental Protection Agency (EPA) in some countries (United States and European Union) but it still widely used in developing countries. Because of its use as a pesticide, the possibility that this herbicide could be an environmental contributor to the etiology of PD has received a great deal attention. Furthermore, epidemiological studies have suggested an increased incidence of PD 
associated with PQ exposure, raising the possibility that PQ could be an environmental parkinsonian toxin. It has been reported several cases of lethal poisoning resulting from ingestion or skin contact and this is the main route of exposure of workers from prolonged contact. Experimental studies using PQ showed that acute exposure to this pesticide had deleterious effects on lung, liver and kidney, whereas chronic PQ exposure induced Parkinsonism. However, significant damage to the brain is reported in individuals who died from PQ intoxication despite the fact that the ability of PQ to cross the blood-brain barrier spontaneously is limited. Being a charged molecule, PQ enters the brain via the neutral amino acid transporter before $\mathrm{Na}^{+}$-dependent uptake into cells occurs. Based of its structural similarities to $\mathrm{MPP}^{+}$, the mechanism of action of PQ was initially thought to be through selective complex I inhibition. However, complex I blockade does not appear to play a significant role in PQ induced neurotoxicity because this toxin has low affinity to mitochondrial complex I and only at high doses. Interestingly, complex III of mitochondrial respiratory chain is involved in the formation of PQinduced ROS in the brain. At the cytosolic levels, PQ causes cellular toxicity via redox cycling. $\mathrm{PQ}$ dication $\left(\mathrm{PQ}^{2+}\right)$ accepts an electron from a reductant to form $\mathrm{PQ}$ monocation $\left(\mathrm{PQ}^{+}\right)$, which then rapidly reacts with $\mathrm{O}_{2}$ - to produce the superoxide radical $\left(\mathrm{O}_{2}^{-}\right)$and regenerate $\mathrm{PQ}^{2+}$. The formation of $\mathrm{O}_{2}^{-}$initiates a series of chain reactions that lead to the generation of ROS. Moreover, PQ produces depletion of reducing equivalents (NADPH and GSH), due to increased oxidation, resulting in the disruption of important biochemical processes. Therefore oxidative stress arises if antioxidant mechanisms to detoxify ROS generated are compromised resulting in DNA, protein and lipid oxidation, ROS is involved in the mechanism by which PQ induces dopaminergic cell death through the activation of proapoptotic signaling pathways. PQ can trigger the sequential activation of JNK, c-Jun and caspase 3 both in vitro and in vivo, induces cytochrome c release and caspase 9 activation, which are preceded by activation of pro-apoptotic Bax and Bak. PQ neurotoxicity has also been reported to require ER stress associated with the activation of the inositol-requiring enzyme 1 (IRE1), apoptosis signal regulating kinase 1 (ASK1) and JNK. Moreover, repeated exposures of mice to the herbicide PQ increase in brain levels of alpha-synuclein and decrease the endogenous levels of DJ-1 in vitro experiments.

\subsection{Enhancers of autophagy as neuroprotectors in PD}

Autophagy is involved in stress-induced adaptation as well as cellular development, differentiation and survival. Regulation of autophagy determines the fate of cells in multiple organs. One of the main concerns of autophagic regulation is the significance of celltype or tissue specificity. Specifically, neurons could be vulnerable to an accumulation of abnormal components such as cytosolic proteins or organelles that are damaged regarding their post-mitotic nature. Therefore, the regulation of neuronal autophagy in a healthy or diseased environment is most likely context-dependent. Neurons differ from other cell types in that they are post-mitotic and highly dependent on the endo-lysosomal pathway for active signaling in the axons and dendrites. Due to these features, neurons require effective protein degradation as a quality control for cell survival, especially under disease conditions for the removal of toxic components. Any alteration of protein degradation can cause the accumulation of abnormal proteins, leading to cellular toxicity and ultimately neurodegeneration. In this sense, increasing evidences suggest that auto- 
phagic deregulation causes accumulation of abnormal proteins or damaged organelles, which is a characteristic of chronic neurodegenerative conditions, such as PD. A viable therapeutic strategy might be to reduce the accumulation of the toxic protein in the cytoplasm. Indeed, promoting the clearance of aggregate-prone proteins via pharmacological induction of autophagy has proved to be a useful mechanism for protecting cells against the toxic effects of these proteins. An additional benefit of autophagy upregulation in models of neurodegenerative diseases is that it seems to protect cells against apoptotic insults. Some evidence also indicates that autophagy might protect cells against necrotic cell death, although this mechanism has not been studied in the context of neurodegeneration. Therapeutic approaches that promote autophagy could, therefore, have two beneficial effects in the context of neurodegenerative diseases; first, they might improve the removal of toxic aggregate-prone proteins from neurons, and second, they could protect neurons from apoptosis.

Autophagy enhancers can be classified in two groups, mTOR dependent and mTOR independent. For this review we will focus in the second.

Which have effect over mTOR dependent autophagy. The very first known drug identified as an autophagy inducer by mTOR pathway is rapamycin, which was already in clinical use for other indications. Rapamycin is a lipophilic macrolide antibiotic originally used as an immunosuppressant. In mammalian cells, rapamycin inhibits the kinase activity of mTOR by forming a complex with the immunophilin FK506-binding protein of $12 \mathrm{kDa}$ (FKBP12). Rapamycin acts specifically on the mTORC1 complex that suppresses autophagy when active.

Which have effect over mTOR independent autophagy. A growing number of little molecules has been show as autophagy inductors by a non canonical (mTOR dependent) pathway. Most of these substances are molecules used for other pharmacological purposes and indications including neurological diseases. We can highlight resveratrol, lithium, trehalose and carbazepine (CBZ).

Resveratrol. Resveratrol (3,5,4-trihydroxystilbene), a type of natural phenol and a phytoalexin, is produced naturally by grapes, mulber-ries, and certain nuts when under attack by pathogens. This powerful anti-oxidant possesses a broad range of bio-logical effects such as neuroprotection, anti-inflammation, and anti-cancer. It especially attenuates neurodegeneration in animal models of Alzheimer's disease and PD associated with the neuronal accumulation of $\beta$-amyloid and alpha-synuclein, respectively. These therapeutic benefits of resveratrol in neuronal disorders are associated with activation of autophagy. A recent study showed that resveratrol protects against rotenone-mediated neurotoxicity in cellular models of PD by autophagy induction. Recently has been described that suppression of AMPK and/or SIRT1 caused decrease of protein level of LC3-II, indicating that AMPK and/or SIRT1 are required in resveratrol mediated autophagy induction. Moreover, suppression of AMPK caused inhibition of SIRT1 activity and attenuated protective effects of resveratrol on rotenone-induced apoptosis, suggesting that AMPK-SIRT1-autophagy pathway plays an important role in the neuroprotection by resveratrol on PD cellular models. 
Lithium. For more than 60 years, lithium has been the standard pharmacological treatment for bipolar disorder (BD), a chronic mental illness characterized by cycling between moods of mania and depression. However, in the last two decades the role of lithium as an effective neuroprotector has emerged. Thus, Lithium treatment inhibits the activation of caspase- 3 in a PI3K-dependent manner and prevents 6-OHDA and MPP+-induced neuronal death. Lithium's ability to deplete free inositol and subsequently decrease IP3 levels was recently identified as a novel route (independent of mTOR) for inducing autophagy. In this sense, in animal models, therapeutic concentrations of lithium have been shown to facilitate clearance of the mutant form of alpha-synuclein, an autophagy substrate. These protective effects suggest that lithium may have substantial therapeutic potential in the treatment of PD.

Trehalose. Trehalose is a nonreducing disaccharide found in organisms from bacteria to plants, including yeast, fungi, and invertebrates. It protects the integrity of cells against various stresses like heat, dehydration, cold, desiccation, and oxidation by preventing protein denaturation, and it is the sugar in the hemolymph of invertebrates. The majority of the protecting properties of trehalose were discovered in yeast ; however, it also has beneficial effects in mammals where it is not endogenously synthesized. In this sense has been recently identified trehalose as a mTOR-independent autophagy enhancer. Although the exact mechanism by which this drug promotes autophagy is unknown, trehalose at a relatively low concentration disaggregates existent A53T-alpha-synuclein protofibrils and fibrils into random coil or soluble $\beta$-sheet conformers, and trehalose at a higher concentration inhibits the formation of A53T alpha-synuclein fibrils. In addition, by stabilizing the partially unfolded protein or activating autophagy in an mTOR independent manner, trehalose accelerates the clearance of the aggregate-prone proteins in various neurodegenerative disorders including polyglutamine in the R6/2 mouse model of Huntington disease, beta-amyloid in Alzheimer's disease, and alphasynuclein in PD. Finally, trehalose also protected cells against several proapoptotic insults.

Carbamazepine. Carbamazepine (CBZ) is an anticonvulsant and mood-stabilizing drug used primarily in the treatment of epilepsy and bipolar disorder, as well as certain neuralgias. The mechanism of action of CBZ and its derivatives is relatively well understood, voltage-gated sodium channels or potentiation of gamma-aminobutyric acid receptors are implicated. However new effects for CBZ has been described in the last years. Thus, CBZ has been implicated with autophagy induction through inhibition of inositol synthesis, which decreases intracellular levels of IP3. Consistent with a role for inositol depletion in autophagy regulation, CBZ significantly reduced EGFP-HDQ74 aggregates and attenuated polyglutamine toxicity in COS-7 cells and enhanced clearance of A30P alpha-synuclein. CBZ also significantly ameliorated rotenone-induced damage in SH-SY5Y cells, by inhibiting the ROS production and inhibiting neuronal apoptosis, and especially for the clearance of malfunction organelles by autophagy induction. 


\section{Conclusions and future perspectives}

Future studies may focus on identifying specific molecules that modulate each step in the autophagy pathway. Small molecules and pharmacologic agents that can more selectively modulate certain aspects of autophagic stress may also help usher in the first wave of diseasespecific therapies. Ideally, small molecule regulators would affect only certain aspects or targets of the autophagy pathway, since global inhibition or enhancement of protein turnover could be problematic. In situations with substantial aggregation, however, a global induction of autophagy may be required provided this does not outstrip the degradative capacity of the aged or diseased cell. Promoting expression of biomolecules required for both induction and clearance of autophagosomes may serve to prevent potential autophagic stress. To determine the mechanism of the possible neuroprotective role of autophagy in PD would provide, eventually, the design of therapeutic interventions (drugs or cell therapy) for treatment of patients affected by this disease.

\section{Funding sources}

Dr. Rosa A. González-Polo received research support from ISCIII (Ministerio de Economía y Competitividad, Spain (CP0800010, PI11/0040) and FUNDESALUD (PRIS11014). Dr. José M. Fuentes received research support from the ISCIII (Ministerio de Economía y Competitividad, Spain (PI12/02280), FUNDESALUD (PRIS11019), CIBERNED (CB06/05/004) and Consejería, Economía, Comercio e Innovación, Junta de Extremadura (GRU10054). Mireia Niso-Santano was supported as a postdoctoral researcher by the University of Extremadura. Rosa A. González-Polo was supported by a "Miguel Servet" contract (ISCIII, Ministerio de Economía y Competitividad, Spain). Elisa Pizarro-Estrella is supported by a predoctoral contract from CIBERNED. Jose M Bravo San-Pedro and Ruben Gómez-Sánchez are beneficiaries to fellowship from Univesity of Extremadura and Minister of Economia y Competitividad respectively.

\section{Abbreviations}

The following abbreviations were used in this paper:

PD, Parkinson's disease; CBZ, carbamazepine; LB, Lewy bodies; LAMP-2A, Lysosomal-associated membrane protein 2A; LRRK2, Leucine-rich repeat kinase 2; PINK1, PTEN-induced kinase 1; CMA, chaperone-mediated autophagy; mTOR, mammalian target of rapamicyn; FKBP12, FK506-binding protein of 12 kDa; AMPK, 5'-adenosine monophosphate-activated protein kinase; $\mathrm{MPP}^{+}$, 1-methyl-4-phenylpyridinium; MPTP, 1-methyl-4-phenyl-1,2,3,6-tetrahydropyridine; PQ, paraquat; Atg, autophagy genes; ROS, reactive oxygen species; $\mathrm{BP}$, bipolar disorder; LC3, microtubule-associated protein light chain 3; GBA-1, glucocerebrosidase; MAO$\mathrm{B}$, monoamine oxidase B; DAT, dopamine transporter; JNK, c-jun N-terminal kinase JNK; 6- 
OHDA, 6-Hydroxydopamine; UPR, unfolded protein response; IRE1, inositol-requiring enzyme 1; ASK1, apoptosis signal regulating kinase 1; GSH, glutathione

\section{Author details}

Rosa A. González-Polo ${ }^{1}$, Rubén Gómez-Sánchez ${ }^{1}$, Lydia Sánchez-Erviti², José M Bravo-San Pedro ${ }^{1}$, Elisa Pizarro-Estrella ${ }^{1}$, Mireia Niso-Santano ${ }^{1,3}$ and José M. Fuentes ${ }^{1}$

*Address all correspondence to: jfuentes@unex.es, rosapolo@unex.es

1 Centro de Investigación Biomédica en Red sobre Enfermedades Neurodegenerativas (CIBERNED). Departamento de Bioquímica y Biología Molecular y Genética, E. Enfermería y T.O., Universidad de Extremadura, CP 10003, Cáceres, España

2 Department of Clinical Neuroscience, UCL Institute of Neurology, London, UK

3 INSERM, U848, Institut Gustave Roussy, Université Paris Sud, Paris, France

\section{References}

[1] Rodriguez-Oroz, M.C., et al., Initial clinical manifestations of Parkinson's disease: features and pathophysiological mechanisms. Lancet Neurol, 2009. 8(12): p. 1128-39.

[2] Spillantini, M.G., et al., Alpha-synuclein in Lewy bodies. Nature, 1997. 388(6645): p. 839-40.

[3] Polymeropoulos, M.H., et al., Mutation in the alpha-synuclein gene identified in families with Parkinson's disease. Science, 1997. 276(5321): p. 2045-7.

[4] Kruger, R., et al., Ala30Pro mutation in the gene encoding alpha-synuclein in Parkinson's disease. Nat Genet, 1998. 18(2): p. 106-8.

[5] Singleton, A.B., et al., alpha-Synuclein locus triplication causes Parkinson's disease. Science, 2003. 302(5646): p. 841.

[6] Li, J.Y., et al., Lewy bodies in grafted neurons in subjects with Parkinson's disease suggest host-to-graft disease propagation. Nat Med, 2008. 14(5): p. 501-3.

[7] Kordower, J.H., et al., Lewy body-like pathology in long-term embryonic nigral transplants in Parkinson's disease. Nat Med, 2008. 14(5): p. 504-6.

[8] Braak, H., et al., Staging of brain pathology related to sporadic Parkinson's disease. Neurobiol Aging, 2003. 24(2): p. 197-211.

[9] Lee, H.J., S. Patel, and S.J. Lee, Intravesicular localization and exocytosis of alpha-synuclein and its aggregates. J Neurosci, 2005. 25(25): p. 6016-24. 
[10] Desplats, P., et al., Inclusion formation and neuronal cell death through neuron-toneuron transmission of alpha-synuclein. Proc Natl Acad Sci U S A, 2009. 106(31): p. 13010-5.

[11] Vella, L.J., et al., Packaging of prions into exosomes is associated with a novel pathway of PrP processing. J Pathol, 2007. 211(5): p. 582-90.

[12] Alvarez-Erviti, L., et al., Lysosomal dysfunction increases exosome-mediated alphasynuclein release and transmission. Neurobiol Dis, 2011. 42(3): p. 360-7.

[13] Emmanouilidou, E., et al., Cell-produced alpha-synuclein is secreted in a calcium-dependent manner by exosomes and impacts neuronal survival. J Neurosci, 2010. 30(20): p. 6838-51.

[14] Schapira, A.H., et al., Mitochondrial complex I deficiency in Parkinson's disease. J Neurochem, 1990. 54(3): p. 823-7.

[15] Mizuno, Y., et al., Inhibition of ATP synthesis by 1-methyl-4-phenylpyridinium ion $(\mathrm{MPP}+)$ in isolated mitochondria from mouse brains. Neurosci Lett, 1987. 81(1-2): p. 204-8.

[16] Sherer, T.B., et al., Mechanism of toxicity in rotenone models of Parkinson's disease. J Neurosci, 2003. 23(34): p. 10756-64.

[17] Valente, E.M., et al., Hereditary early-onset Parkinson's disease caused by mutations in PINK1. Science, 2004. 304(5674): p. 1158-60.

[18] Kitada, T., et al., Mutations in the parkin gene cause autosomal recessive juvenile parkinsonism. Nature, 1998. 392(6676): p. 605-8.

[19] Matsuda, N., et al., PINK1 stabilized by mitochondrial depolarization recruits Parkin to damaged mitochondria and activates latent Parkin for mitophagy. J Cell Biol, 2010. 189(2): p. 211-21.

[20] Owen, A.D., et al., Oxidative stress and Parkinson's disease. Ann N Y Acad Sci, 1996. 786: p. 217-23.

[21] Kachergus, J., et al., Identification of a novel LRRK2 mutation linked to autosomal dominant parkinsonism: evidence of a common founder across European populations. Am J Hum Genet, 2005. 76(4): p. 672-80.

[22] Goker-Alpan, O., et al., Glucocerebrosidase mutations are an important risk factor for Lewy body disorders. Neurology, 2006. 67(5): p. 908-10.

[23] Neumann, J., et al., Glucocerebrosidase mutations in clinical and pathologically proven Parkinson's disease. Brain, 2009. 132(Pt 7): p. 1783-94.

[24] Yang, Z. and D.J. Klionsky, Eaten alive: a history of macroautophagy. Nat Cell Biol, 2010. 12(9): p. 814-22.

[25] Olson, T.S. and J.F. Dice, Regulation of protein degradation rates in eukaryotes. Curr Opin Cell Biol, 1989. 1(6): p. 1194-200. 
[26] Mortimore, G.E. and A.R. Poso, Intracellular protein catabolism and its control during nutrient deprivation and supply. Annu Rev Nutr, 1987. 7: p. 539-64.

[27] Kiffin, R., et al., Activation of chaperone-mediated autophagy during oxidative stress. Mol Biol Cell, 2004. 15(11): p. 4829-40.

[28] Cadwell, K., et al., A key role for autophagy and the autophagy gene Atg1611 in mouse and human intestinal Paneth cells. Nature, 2008. 456(7219): p. 259-63.

[29] Li, W., Q. Yang, and Z. Mao, Chaperone-mediated autophagy: machinery, regulation and biological consequences. Cell Mol Life Sci, 2011. 68(5): p. 749-63.

[30] Hailey, D.W., et al., Mitochondria supply membranes for autophagosome biogenesis during starvation. Cell, 2010. 141(4): p. 656-67.

[31] Ravikumar, B., et al., Plasma membrane contributes to the formation of pre-autophagosomal structures. Nat Cell Biol, 2010. 12(8): p. 747-57.

[32] Ravikumar, B., et al., Mammalian macroautophagy at a glance. J Cell Sci, 2009. 122(Pt 11): p. 1707-11.

[33] Xie, Z. and D.J. Klionsky, Autophagosome formation: core machinery and adaptations. Nat Cell Biol, 2007. 9(10): p. 1102-9.

[34] Dennis, P.B., S. Fumagalli, and G. Thomas, Target of rapamycin (TOR): balancing the opposing forces of protein synthesis and degradation. Curr Opin Genet Dev, 1999. 9(1): p. $49-54$.

[35] Hosokawa, N., et al., Nutrient-dependent mTORC1 association with the ULK1-Atg13FIP200 complex required for autophagy. Mol Biol Cell, 2009. 20(7): p. 1981-91.

[36] Scott, S.V., et al., Apg13p and Vac8p are part of a complex of phosphoproteins that are required for cytoplasm to vacuole targeting. J Biol Chem, 2000. 275(33): p. 25840-9.

[37] Mijaljica, D., M. Prescott, and R.J. Devenish, Microautophagy in mammalian cells: revisiting a 40-year-old conundrum. Autophagy, 2011. 7(7): p. 673-82.

[38] Chiang, H.L., et al., A role for a 70-kilodalton heat shock protein in lysosomal degradation of intracellular proteins. Science, 1989. 246(4928): p. 382-5.

[39] Cuervo, A.M. and J.F. Dice, A receptor for the selective uptake and degradation of proteins by lysosomes. Science, 1996. 273(5274): p. 501-3.

[40] Anglade, P., et al., Apoptosis and autophagy in nigral neurons of patients with Parkinson's disease. Histol Histopathol, 1997. 12(1): p. 25-31.

[41] Dehay, B., et al., Pathogenic lysosomal depletion in Parkinson's disease. J Neurosci, 2010. 30(37): p. 12535-44.

[42] Alvarez-Erviti, L., et al., Chaperone-mediated autophagy markers in Parkinson disease brains. Arch Neurol, 2010. 67(12): p. 1464-72. 
[43] Cuervo, A.M., et al., Impaired degradation of mutant alpha-synuclein by chaperonemediated autophagy. Science, 2004. 305(5688): p. 1292-5.

[44] Mak, S.K., et al., Lysosomal degradation of alpha-synuclein in vivo. J Biol Chem, 2010. 285(18): p. 13621-9.

[45] Martinez-Vicente, M., et al., Dopamine-modified alpha-synuclein blocks chaperonemediated autophagy. J Clin Invest, 2008. 118(2): p. 777-88.

[46] Narendra, D., et al., Parkin is recruited selectively to impaired mitochondria and promotes their autophagy. J Cell Biol, 2008. 183(5): p. 795-803.

[47] Narendra, D.P., et al., PINK1 is selectively stabilized on impaired mitochondria to activate Parkin. PLoS Biol, 2010. 8(1): p. e1000298.

[48] Dehay, B., et al., Loss of P-type ATPase ATP13A2/PARK9 function induces general lysosomal deficiency and leads to Parkinson disease neurodegeneration. Proc Natl Acad Sci U S A, 2012. 109(24): p. 9611-6.

[49] Sanchez-Danes, A., et al., Disease-specific phenotypes in dopamine neurons from human iPS-based models of genetic and sporadic Parkinson's disease. EMBO Mol Med, 2012. 4(5): p. 380-95.

[50] Bravo-San Pedro, J.M., et al., The LRRK2 G2019S mutant exacerbates basal autophagy through activation of the MEK/ERK pathway. Cell Mol Life Sci, 2012.

[51] Good, P.F., et al., Protein nitration in Parkinson's disease. J Neuropathol Exp Neurol, 1998. 57(4): p. 338-42.

[52] Dexter, D.T., et al., Basal lipid peroxidation in substantia nigra is increased in Parkinson's disease. J Neurochem, 1989. 52(2): p. 381-9.

[53] Dexter, D.T., et al., Increased levels of lipid hydroperoxides in the parkinsonian substantia nigra: an HPLC and ESR study. Mov Disord, 1994. 9(1): p. 92-7.

[54] Perry, T.L., D.V. Godin, and S. Hansen, Parkinson's disease: a disorder due to nigral glutathione deficiency? Neurosci Lett, 1982. 33(3): p. 305-10.

[55] Kish, S.J., C. Morito, and O. Hornykiewicz, Glutathione peroxidase activity in Parkinson's disease brain. Neurosci Lett, 1985. 58(3): p. 343-6.

[56] Yamin, G., V.N. Uversky, and A.L. Fink, Nitration inhibits fibrillation of human alphasynuclein in vitro by formation of soluble oligomers. FEBS Lett, 2003. 542(1-3): p. 147-52.

[57] Paxinou, E., et al., Induction of alpha-synuclein aggregation by intracellular nitrative insult. J Neurosci, 2001. 21(20): p. 8053-61.

[58] Langston, J.W. and P.A. Ballard, Jr., Parkinson's disease in a chemist working with 1methyl-4-phenyl-1,2,5,6-tetrahydropyridine. N Engl J Med, 1983. 309(5): p. 310.

[59] Davis, G.C., et al., Chronic Parkinsonism secondary to intravenous injection of meperidine analogues. Psychiatry Res, 1979. 1(3): p. 249-54. 
[60] Riachi, N.J., J.C. LaManna, and S.I. Harik, Entry of 1-methyl-4-phenyl-1,2,3,6-tetrahydropyridine into the rat brain. J Pharmacol Exp Ther, 1989. 249(3): p. 744-8.

[61] Chiba, K., A. Trevor, and N. Castagnoli, Jr., Metabolism of the neurotoxic tertiary amine, MPTP, by brain monoamine oxidase. Biochem Biophys Res Commun, 1984. 120(2): p. 574-8.

[62] Javitch, J.A., et al., Parkinsonism-inducing neurotoxin, N-methyl-4-phenyl-1,2,3,6 -tetrahydropyridine: uptake of the metabolite $\mathrm{N}$-methyl-4-phenylpyridine by dopamine neurons explains selective toxicity. Proc Natl Acad Sci U S A, 1985. 82(7): p. 2173-7.

[63] Gonzalez-Polo, R.A., et al., Mechanisms of $\mathrm{MPP}(+)$ incorporation into cerebellar granule cells. Brain Res Bull, 2001. 56(2): p. 119-23.

[64] Sheldon, A.L. and M.B. Robinson, The role of glutamate transporters in neurodegenerative diseases and potential opportunities for intervention. Neurochem Int, 2007. 51(6-7): p. 333-55.

[65] Nicklas, W.J., I. Vyas, and R.E. Heikkila, Inhibition of NADH-linked oxidation in brain mitochondria by 1-methyl-4-phenyl-pyridine, a metabolite of the neurotoxin, 1-methyl-4-phenyl-1,2,5,6-tetrahydropyridine. Life Sci, 1985. 36(26): p. 2503-8.

[66] Di Monte, D., et al., 1-Methyl-4-phenyl-1,2,3,6-tetrahydropyridine (MPTP) and 1-methyl-4-phenylpyridine (MPP+) cause rapid ATP depletion in isolated hepatocytes. Biochem Biophys Res Commun, 1986. 137(1): p. 310-5.

[67] Saporito, M.S., B.A. Thomas, and R.W. Scott, MPTP activates c-Jun NH(2)-terminal kinase (JNK) and its upstream regulatory kinase MKK4 in nigrostriatal neurons in vivo. J Neurochem, 2000. 75(3): p. 1200-8.

[68] Karunakaran, S., et al., Selective activation of p38 mitogen-activated protein kinase in dopaminergic neurons of substantia nigra leads to nuclear translocation of p53 in 1methyl-4-phenyl-1,2,3,6-tetrahydropyridine-treated mice. J Neurosci, 2008. 28(47): p. 12500-9.

[69] Hassouna, I., et al., Increase in bax expression in substantia nigra following 1-methyl-4phenyl-1,2,3,6-tetrahydropyridine (MPTP) treatment of mice. Neurosci Lett, 1996. 204(1-2): p. 85-8.

[70] Vila, M., et al., Bax ablation prevents dopaminergic neurodegeneration in the 1-methyl4-phenyl-1,2,3,6-tetrahydropyridine mouse model of Parkinson's disease. Proc Natl Acad Sci U S A, 2001. 98(5): p. 2837-42.

[71] McCormack, A.L., et al., Pathologic modifications of alpha-synuclein in 1-methyl-4phenyl-1,2,3,6-tetrahydropyridine (MPTP)-treated squirrel monkeys. J Neuropathol Exp Neurol, 2008. 67(8): p. 793-802.

[72] Wang, C., et al., Stress-induced alterations in parkin solubility promote parkin aggregation and compromise parkin's protective function. Hum Mol Genet, 2005. 14(24): p. 3885-97. 
[73] Talpade, D.J., et al., In vivo labeling of mitochondrial complex I (NADH:ubiquinone oxidoreductase) in rat brain using [(3)H]dihydrorotenone. J Neurochem, 2000. 75(6): p. 2611-21.

[74] Chung, W.G., C.L. Miranda, and C.S. Maier, Epigallocatechin gallate (EGCG) potentiates the cytotoxicity of rotenone in neuroblastoma SH-SY5Y cells. Brain Res, 2007. 1176: p. 133-42.

[75] Wang, X.F., et al., Inhibitory effects of pesticides on proteasome activity: implication in Parkinson's disease. Neurobiol Dis, 2006. 23(1): p. 198-205.

[76] Sherer, T.B., et al., An in vitro model of Parkinson's disease: linking mitochondrial impairment to altered alpha-synuclein metabolism and oxidative damage. J Neurosci, 2002. 22(16): p. 7006-15.

[77] Jonsson, G. and C. Sachs, Actions of 6-hydroxydopamine quinones on catecholamine neurons. J Neurochem, 1975. 25(4): p. 509-16.

[78] Ungerstedt, U., 6-Hydroxy-dopamine induced degeneration of central monoamine neurons. Eur J Pharmacol, 1968. 5(1): p. 107-10.

[79] Blum, D., et al., Molecular pathways involved in the neurotoxicity of 6-OHDA, dopamine and MPTP: contribution to the apoptotic theory in Parkinson's disease. Prog Neurobiol, 2001. 65(2): p. 135-72.

[80] Luthman, J., et al., Selective lesion of central dopamine or noradrenaline neuron systems in the neonatal rat: motor behavior and monoamine alterations at adult stage. Behav Brain Res, 1989. 33(3): p. 267-77.

[81] Kienzl, E., et al., The role of transition metals in the pathogenesis of Parkinson's disease. J Neurol Sci, 1995. 134 Suppl: p. 69-78.

[82] Linert, W., et al., Dopamine, 6-hydroxydopamine, iron, and dioxygen--their mutual interactions and possible implication in the development of Parkinson's disease. Biochim Biophys Acta, 1996. 1316(3): p. 160-8.

[83] Deumens, R., A. Blokland, and J. Prickaerts, Modeling Parkinson's disease in rats: an evaluation of 6-OHDA lesions of the nigrostriatal pathway. Exp Neurol, 2002. 175(2): p. 303-17.

[84] Blandini, F., M.T. Armentero, and E. Martignoni, The 6-hydroxydopamine model: news from the past. Parkinsonism Relat Disord, 2008. 14 Suppl 2: p. S124-9.

[85] Oestreicher, E., et al., Degeneration of nigrostriatal dopaminergic neurons increases iron within the substantia nigra: a histochemical and neurochemical study. Brain Res, 1994. 660(1): p. 8-18.

[86] Glinka, Y., M. Gassen, and M.B. Youdim, Mechanism of 6-hydroxydopamine neurotoxicity. J Neural Transm Suppl, 1997. 50: p. 55-66. 
[87] Holtz, W.A., et al., Oxidative stress-triggered unfolded protein response is upstream of intrinsic cell death evoked by parkinsonian mimetics. J Neurochem, 2006. 99(1): p. 54-69.

[88] Ryu, E.J., et al., Endoplasmic reticulum stress and the unfolded protein response in cellular models of Parkinson's disease. J Neurosci, 2002. 22(24): p. 10690-8.

[89] Drechsel, D.A. and M. Patel, Role of reactive oxygen species in the neurotoxicity of environmental agents implicated in Parkinson's disease. Free Radic Biol Med, 2008. 44(11): p. 1873-86.

[90] Cannon, J.R. and J.T. Greenamyre, Neurotoxic in vivo models of Parkinson's disease recent advances. Prog Brain Res, 2010. 184: p. 17-33.

[91] Liou, H.H., et al., Environmental risk factors and Parkinson's disease: a case-control study in Taiwan. Neurology, 1997. 48(6): p. 1583-8.

[92] Smith, J.G., Paraquat poisoning by skin absorption: a review. Hum Toxicol, 1988. 7(1): p. 15-9.

[93] Dinis-Oliveira, R.J., et al., Paraquat exposure as an etiological factor of Parkinson's disease. Neurotoxicology, 2006. 27(6): p. 1110-22.

[94] Grant, H., P.L. Lantos, and C. Parkinson, Cerebral damage in paraquat poisoning. Histopathology, 1980. 4(2): p. 185-95.

[95] Shimizu, K., et al., Carrier-mediated processes in blood--brain barrier penetration and neural uptake of paraquat. Brain Res, 2001. 906(1-2): p. 135-42.

[96] Richardson, J.R., et al., Paraquat neurotoxicity is distinct from that of MPTP and rotenone. Toxicol Sci, 2005. 88(1): p. 193-201.

[97] Castello, P.R., D.A. Drechsel, and M. Patel, Mitochondria are a major source of paraquat-induced reactive oxygen species production in the brain. J Biol Chem, 2007. 282(19): p. 14186-93.

[98] Miller, G.W., Paraquat: the red herring of Parkinson's disease research. Toxicol Sci, 2007. 100(1): p. 1-2.

[99] Moretto, A. and C. Colosio, Biochemical and toxicological evidence of neurological effects of pesticides: the example of Parkinson's disease. Neurotoxicology, 2011. 32(4): p. 383-91.

[100] Bus, J.S. and J.E. Gibson, Paraquat: model for oxidant-initiated toxicity. Environ Health Perspect, 1984. 55: p. 37-46.

[101] Ryter, S.W., et al., Mechanisms of cell death in oxidative stress. Antioxid Redox Signal, 2007. 9(1): p. 49-89.

[102] West, J.D. and L.J. Marnett, Endogenous reactive intermediates as modulators of cell signaling and cell death. Chem Res Toxicol, 2006. 19(2): p. 173-94. 
[103] Peng, J., et al., The herbicide paraquat induces dopaminergic nigral apoptosis through sustained activation of the JNK pathway. J Biol Chem, 2004. 279(31): p. 32626-32.

[104] Niso-Santano, M., et al., Low concentrations of paraquat induces early activation of extracellular signal-regulated kinase $1 / 2$, protein kinase $\mathrm{B}$, and c-Jun $\mathrm{N}$-terminal kinase $1 / 2$ pathways: role of c-Jun $\mathrm{N}$-terminal kinase in paraquat-induced cell death. Toxicol Sci, 2006. 92(2): p. 507-15.

[105] Fei, Q., et al., Paraquat neurotoxicity is mediated by a Bak-dependent mechanism. J Biol Chem, 2008. 283(6): p. 3357-64.

[106] Gonzalez-Polo, R.A., et al., Paraquat-induced apoptotic cell death in cerebellar granule cells. Brain Res, 2004. 1011(2): p. 170-6.

[107] Yang, W. and E. Tiffany-Castiglioni, Paraquat-induced apoptosis in human neuroblastoma SH-SY5Y cells: involvement of p53 and mitochondria. J Toxicol Environ Health A, 2008. 71(4): p. 289-99.

[108] Yang, W., et al., Paraquat activates the IRE1/ASK1/JNK cascade associated with apoptosis in human neuroblastoma SH-SY5Y cells. Toxicol Lett, 2009. 191(2-3): p. 203-10.

[109] Niso-Santano, M., et al., Activation of apoptosis signal-regulating kinase 1 is a key factor in paraquat-induced cell death: modulation by the Nrf2/Trx axis. Free Radic Biol Med, 2010. 48(10): p. 1370-81.

[110] Manning-Bog, A.B., et al., The herbicide paraquat causes up-regulation and aggregation of alpha-synuclein in mice: paraquat and alpha-synuclein. J Biol Chem, 2002. 277(3): p. 1641-4.

[111] Gonzalez-Polo, R., et al., Silencing DJ-1 reveals its contribution in paraquat-induced autophagy. J Neurochem, 2009. 109(3): p. 889-98.

[112] Fleming, A., et al., Chemical modulators of autophagy as biological probes and potential therapeutics. Nat Chem Biol, 2011. 7(1): p. 9-17.

[113] Ravikumar, B., et al., Inhibition of mTOR induces autophagy and reduces toxicity of polyglutamine expansions in fly and mouse models of Huntington disease. Nat Genet, 2004. 36(6): p. 585-95.

[114] Ravikumar, B., et al., Rapamycin pre-treatment protects against apoptosis. Hum Mol Genet, 2006. 15(7): p. 1209-16.

[115] Kim, D.H., et al., mTOR interacts with raptor to form a nutrient-sensitive complex that signals to the cell growth machinery. Cell, 2002. 110(2): p. 163-75.

[116] Sarbassov, D.D., et al., Prolonged rapamycin treatment inhibits mTORC2 assembly and Akt/PKB. Mol Cell, 2006. 22(2): p. 159-68.

[117] Walle, T., Bioavailability of resveratrol. Ann N Y Acad Sci, 2011. 1215: p. 9-15.

[118] Richard, T., et al., Neuroprotective properties of resveratrol and derivatives. Ann N Y Acad Sci, 2011. 1215: p. 103-8. 
[119] Tili, E. and J.J. Michaille, Resveratrol, MicroRNAs, Inflammation, and Cancer. J Nucleic Acids, 2011. 2011: p. 102431.

[120] Vingtdeux, V., et al., AMP-activated protein kinase signaling activation by resveratrol modulates amyloid-beta peptide metabolism. J Biol Chem, 2010. 285(12): p. 9100-13.

[121] Wu, Y., et al., Resveratrol-activated AMPK/SIRT1/autophagy in cellular models of Parkinson's disease. Neurosignals, 2011. 19(3): p. 163-74.

[122] Manji, H.K. and R.H. Lenox, Lithium: a molecular transducer of mood-stabilization in the treatment of bipolar disorder. Neuropsychopharmacology, 1998. 19(3): p. 161-6.

[123] King, T.D., G.N. Bijur, and R.S. Jope, Caspase-3 activation induced by inhibition of mitochondrial complex I is facilitated by glycogen synthase kinase-3beta and attenuated by lithium. Brain Res, 2001. 919(1): p. 106-14.

[124] Chen, G., et al., Glycogen synthase kinase 3beta (GSK3beta) mediates 6-hydroxydopamine-induced neuronal death. FASEB J, 2004. 18(10): p. 1162-4.

[125] Sarkar, S., et al., Lithium induces autophagy by inhibiting inositol monophosphatase. J Cell Biol, 2005. 170(7): p. 1101-11.

[126] Sarkar, S. and D.C. Rubinsztein, Inositol and IP3 levels regulate autophagy: biology and therapeutic speculations. Autophagy, 2006. 2(2): p. 132-4.

[127] Chen, Q. and G.G. Haddad, Role of trehalose phosphate synthase and trehalose during hypoxia: from flies to mammals. J Exp Biol, 2004. 207(Pt 18): p. 3125-9.

[128] Kandror, O., et al., Yeast adapt to near-freezing temperatures by STRE/Msn2,4-dependent induction of trehalose synthesis and certain molecular chaperones. Mol Cell, 2004. 13(6): p. 771-81.

[129] Yu, W.B., et al., Trehalose inhibits fibrillation of A53T mutant alpha-synuclein and disaggregates existing fibrils. Arch Biochem Biophys, 2012. 523(2): p. 144-50.

[130] Sarkar, S., et al., Trehalose, a novel mTOR-independent autophagy enhancer, accelerates the clearance of mutant huntingtin and alpha-synuclein. J Biol Chem, 2007. 282(8): p. 5641-52.

[131] Casarejos, M.J., et al., The accumulation of neurotoxic proteins, induced by proteasome inhibition, is reverted by trehalose, an enhancer of autophagy, in human neuroblastoma cells. Neurochem Int, 2011. 58(4): p. 512-20.

[132] Kruger, U., et al., Autophagic degradation of tau in primary neurons and its enhancement by trehalose. Neurobiol Aging, 2011.

[133] Chen, W., et al., Trehalose protects against ocular surface disorders in experimental murine dry eye through suppression of apoptosis. Exp Eye Res, 2009. 89(3): p. 311-8.

[134] Granger, P., et al., Modulation of the gamma-aminobutyric acid type A receptor by the antiepileptic drugs carbamazepine and phenytoin. Mol Pharmacol, 1995. 47(6): p. 1189-96. 
[135] Xiong, N., et al., Potential autophagy enhancers attenuate rotenone-induced toxicity in SH-SY5Y. Neuroscience, 2011. 199: p. 292-302. 
